\title{
The Analysis of Bank Mobile Payment Project's Schedule Management Based on Improved Critical Chain Method
}

\author{
Peng Cheng* \\ International Business School \\ Yunnan University of Finance and Economics \\ Kunming, China
}

\author{
Song Liang \\ Xishan District Rural Credit Cooperatives \\ Kunming, China \\ 50665929@qq.com
}

\begin{abstract}
Bank's mobile payment project schedule management is a crucial part in project management. It is not only related with the development cost of the project but also affects the cost of benefit in the late stage of the project. It is a crucial guarantee to grasp the first opportunity of the market. The core concept of this paper is to pre-pose the risk and retro-pose the idleness. The critical path of the project will be clarified from the perspective of resource constraint. Parallel strategy will be adopted for non-critical procedures and the total working duration will be shortened. At the same time, cutting and pasting method of traditional buffering setting method and the limitations of root variance method are discussed. The improvement concept is proposed, which is skillfully embed analytic hierarchy process and grey evaluation method during the research process of critical chain method. According to the analysis and evaluation to the factors influencing the process and schedule of the project, the safety time of each procedure is intercepted relatively independently according to the best interception percentage as the sub-buffering. All sub-buffering on the whole critical path will be added as the project's buffering.
\end{abstract}

Keywords-project schedule management; critical Chain method; buffering area

\section{INTRODUCTION}

At present, the bank's mobile business is flourishing in China. The convenient and effective operation environment provides good experience for the users and this business has attracted a great number of users. The bank's mobile payment project represents mainstream development trend of "Internet + Finance". At present, there have been more than 70 countries has developed bank's mobile payment projects in the world with Japan as the representative. In Japan, the core of the application of mobile payment project is the telecommunication operator who makes payment products. The mode of cooperation and holding by several large operators is applied in USA. China is unwilling to lag behind. At present, the bank industry in China collaborates together, and is ready to develop diversified bank's mobile payment projects. The statistics shows there have been 70 banks in China who have implemented free transferring service through E-bank and mobile banking at present in order to attract more customers.
The development of new projects is usually required to have short cycle, high efficiency and low cost in order to show competitive advantage in the industry, which is also the core appeal of modern management. The Rural Credit Cooperative also starts to set about the development of these projects during the process, including large projects and special small projects. Objectively speaking, the Rural Credit Cooperative starts later than other commercial banks in the development of bank's mobile payment project. The Rural Credit Cooperative is immature in accumulated experience and many details in the development process need to be explored. If the control is not proper in the project implementation process, it might lead to schedule overdue , budget overrunning and so on. Based on above, this research select a Rural Credit Cooperative planning to develop mobile payment project. I need to carry out investigation and fully clear up the resource reserve status in the early stage of project development, cooperative units of development, development schedule arrangement and possible accompanying risks during the development process in order to give optimization management to the controllability of the project's schedule development.

\section{LITERATURE REVIEW}

Project schedule management is an important part in project management while project management is a must to ensure the project to be completed on time with good quality under objective conditions (Brown,2003)[1]. Project schedule management is an important component of project management process, and it is an essential key as well as basis to realize follow-up management of the project, such as cost and benefit management (Arahanm, 2010)[2]. Much stricter monitoring and control is carried out to the time of project's procedure through a serial of technical methods, ensuring the project to be the best in time dimension during direct process of working duration. It experiences long development from appearance to the perfection of the theory. All these are crucial theoretical research in project schedule management. The research by Anbari (2003)[3] and Joanna (2016) [4]shows with the continuously increasing of project types, expansion of project scale and especially possible integration of new elements in some projects, the managers usually fail to find reference system during management process. Without a referential case or method, the occurrence of these uncertain

* Corresponding author 
problems increases difficulty of project schedule management. The problems existing in management process start to be prominent with the increasing of management difficulty. It is obvious that it cannot meet the requirements of modern project schedule management by simply relying on traditional technical methods. The thinking concept and analytical tool need to be innovated urgently. Therefore, some scholars re-processed traditional theories in recent years and perfected some research details. In addition, some new research tools are inferred based on previous research. These tools have stronger pertinence and accuracy in researching some concrete problem, new perception arising gradually in management concept Aiken, M (1995) [5] and other scholars point out some wrong perception in the past project schedule management. They point out traditional project schedule management concept is bounded, over emphasizing plan while ignoring practice. The project schedule management method must be drawn together with real practice and the plan conclusion must be inspected by case. Otherwise, the analysis result fails to know practice and even might lead to negative influence and loss of great operation time of the project .

The occurrence of above problems fully reveals the analytical concept and analytical tool of traditional project schedule management has fully lost original advantages at present and failed to follow up with the requirement of era. In addition, with increasing difficulty in schedule management in innovation aspect, many existing improvement concepts and innovation methods are still not perfect. Even for relatively scientific methods, other analysis tools should be integrated in proper time according to research demands during the research process of practical issues. In other words, several tools will be applied at the same time to solve the same problem. Such improvement concept is more favorable to demonstration (Chu, C.W. et al, 2012) [6]. At present, project schedule management needs new concept and new methods urgently. It is better to make more branches and find out the most applicable management method for its project field during the research process of different fields (Al-jibouri, 2012)[7].

\section{Establishing DeVElopMent SCHEDUle CONTROL -Management Model for Mobile PAyMent Project}

\section{A. General Modelling Concept}

This paper is selected as the critical chain paper core processing technology, the use of this method to analysis the project schedule study time optimal allocation of resources management issues. The core concept is to ensure optimal time arrangement of whole project instead of optimal time arrangement of partial. Uncertain factors can be added in project schedule plan in order to analyze the influence of these uncertain factors to procedure duration. In addition, the intercepted time can be set to buffer area by cutting down safe time of procedures. This paper combines specific practice of development of bank's mobile payment project and designs applicable model so as to improve the development efficiency of the project.

\section{B. Estimation of Procedure Duration}

Procedure duration usually can be divided into three types during the execution process of project. The first type is flexible time. This kind of time is usually judged by subjective identification of the project executor, which will be greatly disturbed by external environment with significant uncertainty. The second type is rigid time. This kind of time is decided by time demanded inside techniques of the project, which reflects necessary working duration of some procedure. Such kind of time is relatively steady. The third type is time beside control, which is independent of project while associated with the project. For example, all kinds of approval processes in the early stage of the project consume this kind of time. The scholars hope to find out an effective management method to shorten project duration. At the same time, the project duration should not be influenced by excessive shrinkage of duration. Therefore, scholars wish to find a way to balance. The advocator of critical chain method proposes to use $50 \%$ project completion probability of procedure duration to replace estimated duration of $90 \%$ project completion probability. In order to realize such assumption, the most critical first step is to judge duration of all procedures in project, so that the practice can be optimized according to these judgments in the future.

Three estimated durations are assumed to exist in a procedure as follows.

$$
t_{i}=\frac{1}{2}\left(\frac{a+2 c}{3}+\frac{b+2 c}{3}\right)=\frac{a+4 c+b}{6}
$$

Where $\mathrm{a}$ is the least duration, $\mathrm{b}$ is the maximum duration, $\mathrm{c}$ is the possible duration.

\section{Determining Critical Path}

\section{1) hypothesis parameter}

Before determining critical path, the time parameter of procedures should be assumed at first.

The first is earliest starting time ESi. All procedures are strictly linked and their arrangement sequence is logic. Some procedure can be started normally when previous procedure is completed. If previous procedure is completed smoothly, the following procedure can be started at the earliest starting time.

The second is earliest finishing time EFi. This indicator can be measured indirectly and obtained by adding duration of the procedure and earliest starting time of procedure. It reflects optimization of procedure execution condition.

The third is the latest starting time LSi. If the procedure is influenced by factors of previous cycle, postponement will occur. The latest starting time can be obtained by corresponding with maximum postponement limit.

The fourth is the latest finishing time LFi, which can be obtained by latest starting time of the project and procedure duration.

The fifth is total float TFi. In the premise when the total project duration is not influenced, the maximum time interval that procedure $\mathrm{i}$ can be postponed is called total float. 


\section{Identifying Critical Path}

The longest path is $\mu$ i of procedure $i$, and the length of critical path is $\mu$. The difference between above two factors is called total float of procedure i, shown as below.

When $\mathrm{TFi}=0 ; \mu \mathrm{i}=\mu$. The path formed by all procedures is the critical path and all procedures on the path are critical procedures.

\section{Selecting Impact Factor of Project Development Influencing Buffer Area Setting}

With deepening of modern lean management concept, buffer area setting has become a critical cycle involved in critical chain method. It aims at refining the interception of safe time in each step and give concrete analysis to specific circumstance. Therefore, factors which are able to influence the development duration size of bank's mobile payment project should be chosen. After that, sub-buffer of each step should be determined with the help of analytical hierarchical process and grey model. At last, sub-buffers of all steps will be summed up to be confirmed as the final project buffer. Specific steps will be described in details in following chapters.

Selection of Factors Influencing Development of Mobile Payment Project.

The selection of impact factors is the first step to calculate sub-buffer. Selection of different indicators will obtain different buffer area sizes at last. Based on practical research experience by predecessors, combination of qualitative and quantitative will be chosen to influence development duration scale indicators of bank's mobile payment project. After giving a comprehensive consideration to features of project, corresponding secondary indicators will be determined from four aspects of resource factor, project factor, subjective factor and enterprise operation factor.

Resource factor can be subdivided into resource tension degree and resource substitutability. Project factor can be subdivided into technical complexity, location of work procedure and procedure duration. Subjective factor can be subdivided into time invested by managers, risk preference of managers, time invested by participators and skill proficiency of participators. Enterprise operation factor can be subdivided into reward principle, enhancement of technical management capacity and innovation and study ability.

Above all, we can get buffer setting indicator system based on grey evaluation.

\section{1) Determining Indicator Weight}

Similar with contradiction analysis method, all factors influence time of working procedure of the project in different levels. Some have great influence while others have little influence. Therefore, the determination of weight is very crucial. Analytical hierarchical process is the most representative to evaluate the weights of comprehensive factors of qualitative and quantitative. Therefore, the dissertation decides to make use of analytical hierarchical process to determine weights of above indicators based on experts scoring.
Total 12 secondary evaluation indicators are involved in multiple-objective decision-making problem in the development of bank's mobile payment project. Generally speaking, this multi-objective research system is very complicated and it is necessary to give accurate valuation to relative importance of evaluation indicator for such decision-making problem.

\section{E. Buffer Volume Setting Based on Grey Evaluation}

From the indicator system mentioned above, we can get the factor set of factors influencing working procedure $\mathrm{i}$ is $\mathrm{A}$ with corresponding primary indicator system of X1, X2, X3 and $\mathrm{X} 4$. Corresponding secondary indicators are set below $\mathrm{X} 1, \mathrm{X} 2, \mathrm{X} 3$ and $\mathrm{X} 4$.

Evaluation set $\mathrm{V}=$ \{no influence, little influence, ordinary influence, fair influence and great influence $\}$ with evaluation values of $\{0.2,0.4,0.6,0.8,1\}$. Experts are invited to score influential degrees of all factors in the project by questionnaire and tick under option they approve (Please refer to appendix 2). The score to $\mathrm{m}$ impact indicators ugj in procedure $\mathrm{i}$ by expert $\mathrm{K}$ is dgjk, which composes sample matrix.

The evaluation grey white functions on evaluation level 1 for ugj given by each expert is calculated according to sample matrix as follows $\mathrm{fl}(\mathrm{dgj} 1), \mathrm{fl}(\mathrm{dgj} 2), \ldots \mathrm{fl}(\mathrm{dgjk})$. It is used to describe the degree that the evaluation indicator affiliating to some evaluation grey category. Please refer to follows for white function in five levels defining corresponding influential degree.

1. The influencing level is grey category of "no influence", the white function is $\mathrm{f} 1[0,0.2,0.4]$, so we get follows.

$$
f_{1}\left(d_{g j k}\right)=\left\{\begin{array}{cc}
0.2 & d_{g j k} \in[0,0.2] \\
0.4-d_{g j k} & d_{g j k} \in[0.2,0.4] \\
0 & d_{g j k} \notin[0,0.4]
\end{array}\right.
$$

2. The influencing level is grey category of "little influence", the white function is $\mathrm{f} 2[0.2,0.4,0.6]$, so we get follows.

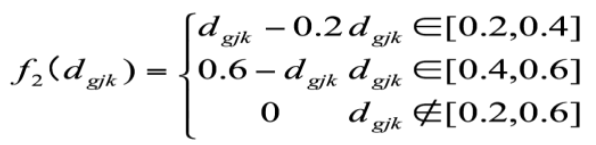

3. The influencing level is grey category of "ordinary influence", the white function is $\mathrm{f3}[0.4,0.6,0.8]$, so we get follows.

$$
f_{3}\left(d_{g j k}\right)=\left\{\begin{array}{c}
d_{g j k}-0.4 d_{g j k} \in[0.4,0.6] \\
0.8-d_{g j k} d_{g j k} \in[0.6,0.8] \\
0 \quad d_{g j k} \notin[0.4,0.8]
\end{array}\right.
$$

4. The influencing level is grey category of "fair influence", the white function is $\mathrm{f} 4[0.6,0.8,1]$, so we get follows. 


$$
f_{4}\left(d_{g j k}\right)=\left\{\begin{array}{cc}
d_{g j k}-0.6 & d_{g j k} \in[0.6,0.8] \\
1-d_{g j k} & d_{g j k} \in[0.8,1] \\
0 & d_{g j k} \notin[0.6,1]
\end{array}\right.
$$

5. The influencing level is grey category of "great influence", the white function is $f 5[0.8,1,+\infty]$. According to grey evaluation theory, we already know secondary evaluation indicator of ugj, experts score of dgjk, white function of $\mathrm{fl}(\mathrm{dgj} 1)$, $\mathrm{fl}(\mathrm{dgj} 2), \ldots \mathrm{fl}(\mathrm{dgjk})$, so the sum-up of white weight that indicator ugj belongs to grey category's.

The weight pgjl reflecting intensity degree that judgement experts believing indicator ugj to affiliate to level 1 is calculated and form grey evaluation weight vector pgj, so we get follows.

$$
p_{g i l}=\frac{w_{g j l}}{\sum_{i=1}^{5} w_{g j l}}=\frac{\sum_{k=1}^{k} f_{1}\left(d_{g j k}\right)}{\sum_{i=1}^{5} \sum_{k=1}^{k} f_{1}\left(d_{g j k}\right)}
$$

After that it can determine grey evaluation weight matrix Pg that indicator Ug corresponding with secondary indicator ugj.

The evaluation grey category and evaluation weight vector of each procedure $i$ in the engineering project will be determined by above steps. Then it carries out comprehensive evaluation and sets buffer through impact factor. Please refer to follows for specific process.

\section{EMPirical analysis of Mobile Payment PRoJect DEVELOPMENT IN RURAL CREDIT COOPERATIVE}

The mobile payment project of Rural Credit Cooperative is jointly developed by Rural Credit Cooperative, Union pay and China Mobile Communication Corporation. The project is planned to be carried out in rural area of Yunnan Province, targeting at improving rural payment environment, enhancing the utilization efficiency of new conceptual product by famers and promote construction of new rural mobile payment. Currently advanced domestic intelligent encryption technique is applied in the project with safe application. The project will link accounts in rural credit cooperative system with settlement system of union pay.

\section{A. Project Implementation Process}

After comprehensive consideration, we can get the project development includes five stages of project preparation stage, project design stage, programming stage, testing stage and acceptance and online stage.

\section{B. Critical Chain Identification of Mobile Payment Project}

\section{1) Determining Project Time and Process}

Specific durations (most possible completion time, most optimistic completion time, most pessimistic completion time) and logic relationship (preceding procedure, retro-posing procedure) of all procedures are involved in the project.

\section{2) Determining Critical Path and Time Parameter}

During the implementation process of rural mobile payment project, the starting of following project needs to rely on completion of previous project. If the schedule of previous project is postponed, the schedule plan of following project will be adjusted accordingly. The following procedures needs to be optimized and adjusted in order to make the project launch online on time due to postponement of previous procedures. Therefore, the critical path of the project should be clarified at first. Flow chart should be made according to logic order of procedure activities to find out the longest path, which is the critical path. Time parameters of all procedures of engineering project should be calculated by traditional project management method and we get the critical path is C-D-A-E-F-H-I-J-K-L-M-P-Q. The project lasts for 484 days.

Remark:

$102+15+11+11+20+31+21+94+51+25+30+30+30+24+10+39$ $+10=484$

\section{A. Determining Indicator Weight \\ 1) Establishing Judgment Matrix}

Experts are invited to score importance of evaluation indicator system for mobile payment project by Rural Credit Cooperative. Total 6 experts are invited, including 2 working in financial departments, 1 working in bank, 1 working in Security Company and other 2 experts engaging in education, who are teachers in university teacher relevant course on management. One expert is a specialist in project technical department of China mobile Communication Corporation of Yunnan Province and one expert is architect in Software Company with years' practical operation experience. It gets mean value from the scores offered by six experts and some mean value is rounded.

\section{2) Determining Combined Weight}

Combined weights of all indicators can be obtained according to all calculated weights of primary indicators and secondary indicators.

\section{B. Determining Buffer by Grey Evaluation Method}

Five experts are invited to score uncertain influence of indicator impact factor to working procedures. Five experts are working in Rural Credit Cooperative and they are professional employees from human resource department, credit management department, general administration department, auditing department and computer department.

Afterwards, the score of influencing degree of uncertainty to procedures offered by experts is taken into white function and then calculate the sum-up of white weights of indicator $u_{g j}$ belonging to grey category of $l$. Then grey evaluation weight matrix $P_{4}$ is obtained.

Primary fuzzy evaluation and fuzzy comprehensive evaluation is carried out to procedures by making use of formulas in Chapter IV and then obtain and determine factors $\varepsilon$ influencing procedures by uncertain factors.

After that, project buffer and feeding buffer size can be calculated as follows. 
$P B=3.3894+1.5963+2.2887+0.4389+0.9968+2.1438+1.65$ $97+7.3109+7.1302+4.0490+1.1805+2.6835+1.2395+1.1007+$ $1.2516+4.9294+1.1290=44.5179$

$F B_{1}=1.5963$

$F B_{2}=1.6597$

$F B_{3}=1.2516$

$F B_{4}=1.1007$

\section{Conclusion And Suggestion}

During case study process by making use of critical chain method, research shows there is indeed non-critical process without constraint of human resource, financial resource, techniques and other resources. If such procedures are connected with other procedures, it will consume extra times. On basis of effective identification of critical chain, we can effectively reduce time consumption on non-critical process, making them carry out with other processes in parallel. In this case, procedures $\mathrm{B}$ and $\mathrm{E}$ are paralleled, procedures $\mathrm{F}$ and $\mathrm{G}$ are paralleled, and procedures $\mathrm{O}, \mathrm{N}$ and $\mathrm{P}$ are paralleled. Procedures $\mathrm{B}, \mathrm{G}, \mathrm{O}$ and $\mathrm{N}$ are non-critical processes, with expecting consumed time of 15 days, 21 days, 20 days and 24 days respectively. These four procedures can be set in the parallel cycle to reduce time consumption. 20 to 40 days are estimated to be reduced. In addition, critical procedures are estimated by making use of expected time of procedures on critical path. Compared to conservative time arrangement, it saves some period. After calculation, research shows the duration of critical chain process estimated by expected duration of procedures is only 484 days, obviously shortened compared to 550 days.

After that, safe time of each procedure is intercepted around critical chain method, postponing solved safe time and setting buffer area of project. Analytical hierarchical process and grey measurement method are integrated in the disposal cycle of such method. The optimal percentage of safe time that can be intersected in each cycle is quantified specifically instead of carrying out unified handling at some fixed percentage by experience. Empirical research shows procedures $\mathrm{A}$ to $\mathrm{Q}$ have been intersected by 3.3894 days, 1.5963 days, 2.2887 days, 0.4389 day, 0.9968 day, 2.1438 days, 1.6597 days, 7.3109 days, 7.1302 days, 4.0490 days, 1.1805 days, 2.6835 days, 1.2395 days, 1.1007 days, 1.2516 days, 4.9294 days and 1.1290 days respectively, 44.5179 days in total. This part of period will be regarded as the project buffer. At this time, if original critical path is calculated by least duration of the project, it takes 371 days. The new critical path takes 426 in total plus 45 days for project buffer. It is shortened by 56 days compared to 484 days previously, reflecting advantages of project operation duration and declining cost expenditure during project development process. Although, the period is shortened by 124 days in total the completion probability is not influenced. Critical chain method has an obvious advantage in project schedule management.

At this time, the buffer area is set precisely in the paper, which is conducive for the manager to control buffer area. Supervision buffer can be carried out at any time according to project schedule, helping managers master the whole project schedule all the time, giving early warning and optimizing management process.

\section{ACKNOWLEDGMENT}

This paper is sponsored by Subject of philosophy and social science of Yunnan Province. Some of the data in this paper were from Xishan District Rural Credit Cooperatives. Therefore, thanks for the information and helps from these cooperatives.

\section{REFERENCES}

[1] I. Brown, "Cell Phone Banking: Predictors of Adoption in South African Exploratory Study," International Journal of Information Management, vol. 85, 2003, pp. 381-394.

[2] S. Arahanm, "Project Management Processes, Methodologies and Economics," Pearson Education South Asia Pte Ltd, vol. 135, 2010, pp. 9-10.

[3] F.T. Anbari, "Earned Value Project Management Method and Extensions," Project Management Journal, vol. 34, 2003, pp. 12-23.

[4] S. Joanna, "The Difficulty in Following Project Schedule as a Key Project Management Challenge: Family Firm Perspective,' Management and Economics Review, vol. 1, 2016, pp. 263-272.

[5] M. Aiken, "Group Decision Sup-port Systems," Review of Business, vol.16, 1995, pp. 38-42.

[6] C.W. Chu, H.L. Ping, "Resource Constrained Project Management Using Enhanced Theory of Constraint," International Journal of Project Management, vol. 20, 2012, pp. 561-567.

[7] S. Al-jibouri, "Effects of Resource Management Regimes on Project Schedule,' International Journal of Project Management, vol. 20, 2012, pp. 271-277.

[8] P. Barber, "Decentralized Site Management: A Case Study," The International Journal of Project Management, vol. 39, 1999, pp. 113-120.

[9] C. Cai, W. Wan, "Critical Chain Management Based on PERT/CPM," China Management Science, vol. 11, 2003, pp. 35-39. 